\title{
Phase separation of a ternary lipid vesicle including $n$-alkane: Rugged vesicle and bilayer flakes formed by separation between highly rigid and flexible domains
}

Cite as: J. Chem. Phys. 150, 064904 (2019); https://doi.org/10.1063/1.5080177

Submitted: 06 November 2018 . Accepted: 22 January 2019. Published Online: 12 February 2019

Mafumi Hishida (D), Ryuta Yanagisawa, Yasuhisa Yamamura (D), and Kazuya Saito (D) 


\title{
Phase separation of a ternary lipid vesicle including $n$-alkane: Rugged vesicle and bilayer flakes formed by separation between highly rigid and flexible domains
}

\author{
Cite as: J. Chem. Phys. 150, 064904 (2019); doi: 10.1063/1.5080177 \\ Submitted: 6 November 2018 - Accepted: 22 January 2019 • \\ Published Online: 12 February 2019
}

Mafumi Hishida, (D) Ryuta Yanagisawa, Yasuhisa Yamamura, (D) and Kazuya Saito ${ }^{\text {a) }}$ (D)

\section{AFFILIATIONS}

Department of Chemistry, Faculty of Pure and Applied Sciences, University of Tsukuba, Tsukuba, Ibaraki 305-8571, Japan

a)Electronic mail: kazuya@chem.tsukuba.ac.jp

\begin{abstract}
We investigate the phase separation of a ternary lipid bilayer including $n$-alkane and construct the ternary phase diagram. When a certain proportion of a long $n$-alkane is mixed with a binary mixture of lipids, which exhibit the disordered liquid-crystalline phase and the ordered gel phase at room temperature, we observed the characteristic morphology of bilayers with phase separation. The ordered bilayer forms flat and rigid domains, which is connected or rimmed with flexible domains in the disordered phase. The asymmetric emergence of the phase separation region close to the ordered phase side is interpreted based on the almost equal distribution of the $n$-alkane to the ordered and disordered phase domains.
\end{abstract}

Published under license by AIP Publishing. https://doi.org/10.1063/1.5080177

\section{INTRODUCTION}

After the suggestion by Simons and Ikonen in 1997, ${ }^{1}$ the inhomogeneity of biomembranes has attracted the attention of many researchers, not only in biology but also in physics and chemistry. The formation of a so-called "raft" domain, ${ }^{2}$ which is the typical inhomogeneity in biomembranes, is considered to be related to many biological functions, such as signal transduction and cooperative membrane trafficking. ${ }^{3}$ Therefore, it is important to understand the formation of a raft and its physical properties. From the physicochemical viewpoint, the formation of a raft is considered to arise from the phase separation of the ordered and disordered phases of lipids. ${ }^{4}$ For example, when a lipid in the ordered gel phase at room temperature [e.g., 1,2-dipalmitoylsn-glycerol-3-phosphocholine (DPPC)] and a lipid in the disordered liquid-crystalline phase at room temperature [e.g., 1,2-dioleoyl-sn-glycero-3-phosphocholine (DOPC)] are mixed, the solid-liquid phase separation is observed at room temperature. ${ }^{5}$
The effect of cholesterol on the phase separation has been widely investigated, ${ }^{5,6}$ since cholesterol is the typical molecule incorporated in real biomembranes and is highly condensed in a raft. ${ }^{2}$ When cholesterol is added to a binary lipid mixture that tends to separate into ordered and disordered phases, like a mixture of DOPC and DPPC, the solid-liquid phase separation changes to liquid-liquid phase separation. ${ }^{5,6}$ The liquid-liquid phase separation seems to be crucial for the formation of a raft in a biomembrane, i.e., the liquid-like domain with cholesterol is considered to be a substructure of a raft. The liquid-like ordered phase, which is called the liquid-ordered phase, is formed by the perturbation of the molecular order in the ordered gel phase induced by cholesterol. ${ }^{7}$

In contrast to the depth and breadth of investigations on the effect of cholesterol, the effects of other organic molecules on the phase separation have, so far, rarely been studied, though various molecules in real biomembranes have specific roles. ${ }^{2}$ The molecules incorporated in a mono-component lipid bilayer have specific effects depending on their molecular 
shapes, ${ }^{8-15}$ which needs further investigation of their effects on the phase separation.

Recently, we reported the effect of tetradecane (TD) on the phase separation. ${ }^{17}$ When TD is mixed with a DOPC/DPPC mixture, a characteristic morphology of the phase-separated vesicle is observed at room temperature. Very rigid and flat DPPC-rich domains in the ordered gel phase are connected by flexible DOPC-rich domains in the disordered liquid-crystalline phase. This morphology is caused by the characteristic effects of TD, which is opposite to the effect of cholesterol. ${ }^{11}$ That is, the molecular arrangement in the ordered gel phase is disordered by cholesterol due to its steric hindrance, but with the addition of $\mathrm{TD}$, the bilayer in the ordered gel phase becomes more ordered and the molecular packing is enhanced. The enhanced packing leads to the rigidification of the bilayer ${ }^{11}$ and also a change in the order-disorder phase transition temperature. ${ }^{10}$ These opposite effects seem to originate from the different molecular shapes of TD and cholesterol, which is bulkier than TD. It is thought that TD fits into the gap in the hydrophobic region of the bilayer because of its linear shape, while cholesterol and other bulky molecules ${ }^{12-14}$ perturb the molecular arrangement in the region.

Our previous report ${ }^{11}$ on the characteristic phase separation dealt with only a single composition of the DOPC/DPPC/TD system. Considering the interesting results, it seems natural to imagine fascinating phase separation behaviors also at other compositions, e.g., domains and vesicles with various morphologies. Also, since various long and linear chain molecules, e.g., other $n$-alkanes and alkanols, have similar effects to TD on the molecular packing and the phase behavior, ${ }^{10,15}$ we would expect that the characteristic morphology of the ternary vesicle observed for the DOPC/DPPC/TD system is common in ternary systems with other long and linear molecules. Thus, in this paper, we report the details of the phase separation, focusing on the ternary phase diagram of the DOPC/DPPC/TD system, and the reproducibility of the characteristic morphology in ternary systems including other $n$-alkanes. $n$-alkanes are known to have an anesthetic effect. ${ }^{16}$ Since the raft behavior was suggested to have some relation to anesthesia, ${ }^{17}$ the present investigation is expected to contribute to the understanding of anesthesia.

\section{MATERIALS AND METHODS}

The following were purchased from Wako Pure Chemical Industries, Ltd::

- DOPC: 1,2-dioleoyl-sn-glycero-3-phosphocholine (>99\%),

- DPPC: 1,2-dipalmitoyl-sn-glycerol-3-phosphocholine (>99\%),

- DLPC: 1,2-dilauroyl-sn-glycero-3-phosphocholine (>99\%),

- DSPC: 1,2-distearoyl-sn-glycero-3-phosphocholine (>99\%).

The following fluorescent reagents were purchased from Avanti Polar Lipids, Inc.:
- Rhodamine-DHPE: rhodamine B 1,2-dihexadecanoylsn-glycero-3-phosphoethanolamine, triethylammonium salt [maximum fluorescence wavelength $580 \mathrm{~nm}$ (red)],

- NBD-PE: N-(7-nitrobenz-2-oxa-1,3-diazol-4-yl)-1,2dihexadecanoyl-sn-glycero-3-phosphoethanolamine, triethylammonium salt [maximum fluorescence wavelength $536 \mathrm{~nm}$ (green)].

Dodecane (DD, >99+\%), tetradecane (TD, >99+\%), and hexadecane (HD, >99+\%) were purchased from Sigma-Aldrich Co. These reagents were used without further purification.

For microscopic observations, each phospholipid (DPPC, DOPC, DLPC, and DSPC) and each alkane (DD, TD, and HD) was dissolved in a chloroform/methanol solution $(2: 1 \mathrm{~V} / \mathrm{V})$ to $10 \mathrm{mM}$, and each fluorescent reagent (rhodamine-DHPE and NBD-PE) was dissolved in the solution to $1 \mu \mathrm{M}$. These solutions were mixed so that the molar fractions were obtained as required. We investigated mainly the DOPC/DPPC/TD system and the complementary DLPC/DSPC/HD and DLPC/DSPC/DD systems. The fluorescent reagent solutions were added to the mixed solutions to make the molar fractions of the fluorescent reagents about 0.1-1 mol. \% against the total amount of lipids. The giant vesicles of the lipid/lipid/alkane systems were prepared by the natural swelling methods. ${ }^{18-20}$ The organic solvent solutions were dropped into a glass tube and only the solvents were evaporated in a vacuum. Pure water was added to the dry lipid/lipid/alkane films so that the total concentration of the lipid/lipid/alkane in water was about $1 \mathrm{mM}$. The aqueous solutions were gently placed in a heat bath for $3.5 \mathrm{~h}$ at about $60^{\circ} \mathrm{C}$. At this temperature, the DPPC (transition temperature $\sim 41^{\circ} \mathrm{C}$ ) is in the liquid-crystalline phase and the lipids and the TD are mixed homogeneously.

For the X-ray diffraction, pure water was added to the above-mentioned lipid/lipid/alkane dry films (without the fluorescent reagents) to make the concentration $100 \mathrm{mM}$. The hydration was performed with sonication at above $50{ }^{\circ} \mathrm{C}$ for over $3 \mathrm{~h}$. In this case, we expected multi-lamellar vesicles to form.

Confocal fluorescence microscopy was performed with an FV1000-D microscope (Olympus). Lasers of wavelength 473 and $559 \mathrm{~nm}$ were used to excite the NBD-PE and rhodamineDHPC, respectively. $40 \times$ and $60 \times$ objective lenses (UPLSAPO $40 \mathrm{X} 2$ and UPLSAPO 60XS, respectively) were used for the observations. All observations were performed at room temperature.

Wide-angle X-ray diffraction (WAXD) was performed at BL10C, Photon Factory, KEK, Japan. The wavelength of the X-rays was $1.488 \AA$, and the sample-to-detector length was about $250 \mathrm{~mm}$, which was calibrated with a standard sample (silver behenate). The detector was a PILATUS3 $200 \mathrm{~K}$. The measurements were performed at room temperature.

\section{RESULTS AND DISCUSSION}

Figure 1(a) shows a confocal microscope image of the phase-separated vesicles with DOPC:DPPC:TD = 40:40:20 (mol:mol:mol), which was reported in our previous paper. ${ }^{11}$ 

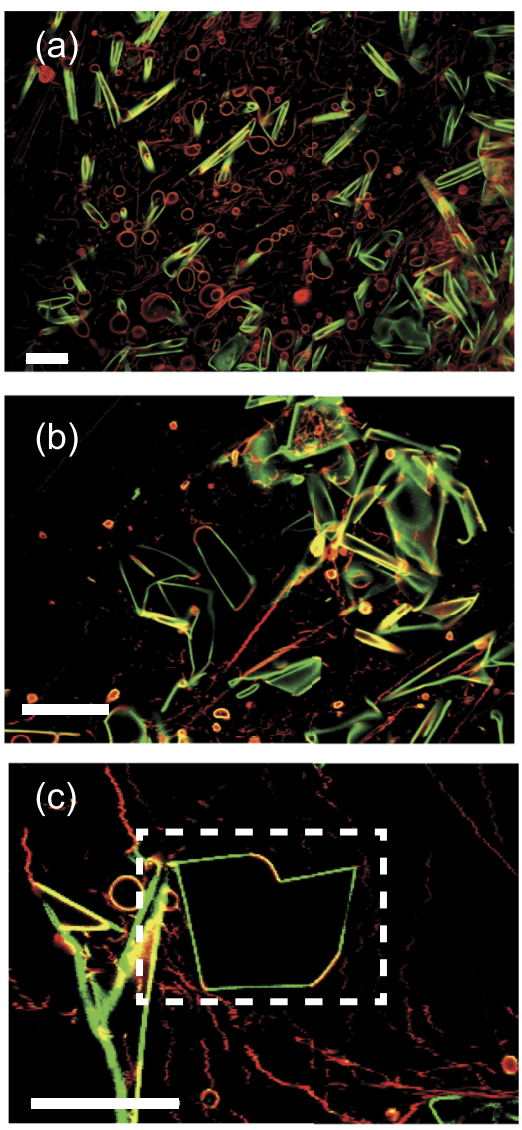

(d)
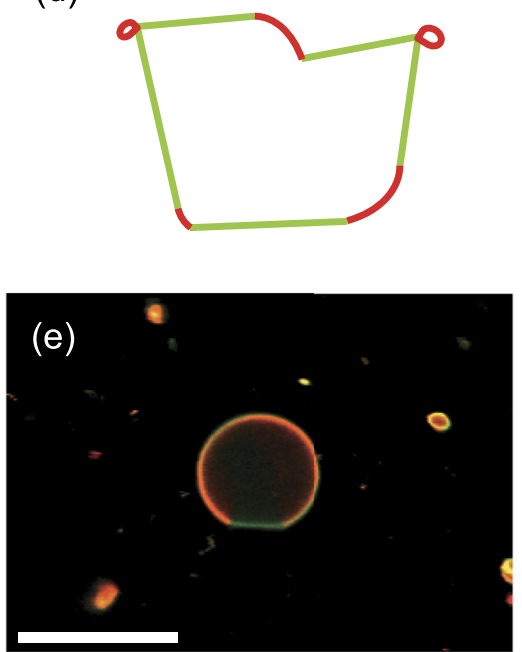

FIG. 1. Confocal microscopy images of the DOPC/DPPC/TD (a)-(c) and DLPC/ DSPC/HD (e) systems showing the characteristic morphology. The green domain is the DPPC-rich (or DSPC-rich) ordered phase, and the red domain is the DOPCrich (or DLPC-rich) disordered phase. Scale bars are $20 \mu \mathrm{m}$. (a) DOPC:DPPC:TD $=40: 40: 20$ (mol:mol:mol) (Reprinted with permission from Hishida et al., J. Chem. Phys. 144, 041103 (2016). Copyright 2016 AIP Publishing LLC.). (b) DOPC:DPPC: $T D=20: 60: 20$. (c) DOPC:DPPC:TD = 33:33:33. (d) Schematic illustration of the vesicle observed in the white square in (c). (e) DLPC:DSPC:HD = 30:30:40.
NBD-PE (green fluorescence) prefers to locate in the ordered phase, while rhodamine-DHPE (red fluorescence) locates in the disordered phase. ${ }^{21}$ Domains in the ordered phase look flat and very rigid, while flexible disordered phase domains connect the flat ordered domains. Similar morphologies were observed in related compositions [for example, DOPC:DPPC:TD $=20: 60: 20$ and 33:33:33 in Figs. 1(b) and 1(c)].

In contrast, in the binary system DOPC/DPPC [Fig. 2(a)], the domain of the ordered phase has a finite curvature and the vesicles are almost spherical. In our previous study, ${ }^{11}$ we showed that a DPPC bilayer with 40 mol. \% of TD is 12 times more rigid than without TD in the gel phase. The rigidification originates from the enhanced molecular packing in the hydrophobic region of the bilayer caused by TD. In the present ternary system consisting of DOPC, DPPC, and TD, the TD is mixed within the DPPC domain and the domain became rigid and flat.

A similar characteristic morphology was also observed in another system, DLPC/DSPC with HD, as shown in Fig. 1(e). In this case, we observed the flat domain of the DSPC-rich gel phase. For the DLPC/DSPC/DD system, on the other hand, such a flat bilayer domain was not observed, and the gel phase domain forms an irregularly bent membrane. As shown in our previous work, ${ }^{10}$ the effect of the alkane depends on its chain length because the molecular packing in the hydrophobic region depends on the contact length between the alkane and an acyl chain of the phospholipid. The enhancement of the packing and the rigidification are weaker with a shorter alkane. ${ }^{10,22}$

At other compositions of DOPC/DPPC/TD, other morphologies were also observed. When the fraction of DOPC is small, flakes of flat membranes, which did not form closed vesicles, were dispersed as shown in Fig. 3. The domains in the disordered phase become located at the rim of the flakes of the ordered phase membrane. Since the fraction of DOPC seems too small to connect the flakes, the closed vesicles shown in Fig. 1 cannot be formed. When the fractions of DOPC and DPPC are quite different, the phase separation was not observed, even if TD was added up to $40 \mathrm{~mol}$. \%. In such cases, spherical
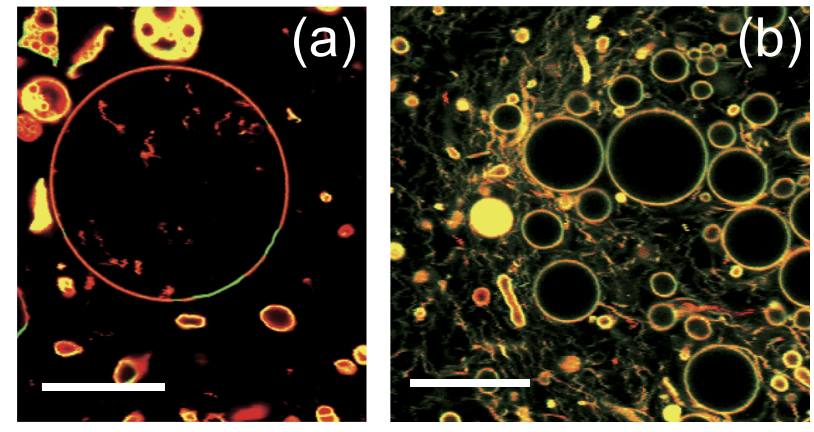

FIG. 2. Confocal microscopy images of the DOPC/DPPC/TD system showing conventional morphology. Scale bars are $20 \mu \mathrm{m}$. (a) DOPC:DPPC:TD $=80: 20: 0$ and (b) DOPC:DPPC:TD = 60:10:30. 


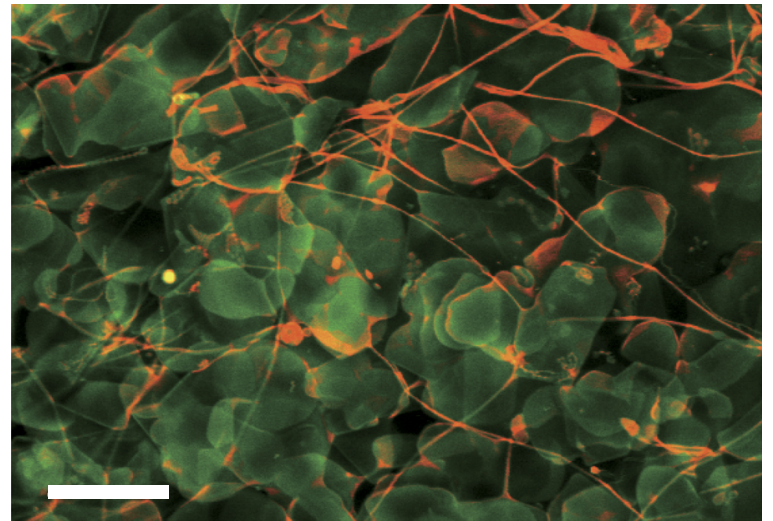

FIG. 3. Confocal microscopy image of the DOPC:DPPC:TD $=10: 60: 30$ system, which does not form vesicles. Scale bar is $20 \mu \mathrm{m}$.

vesicles without phase separation were observed, as shown in Fig. 2(b).

Figure 4 shows the ternary phase diagram for the phase separation of the DOPC/DPPC/TD system at room temperature. When the fraction of TD is more than $40 \mathrm{~mol} . \%$, some of the added TD is excluded from the bilayer and it forms a macro-domain droplet of TD liquid. ${ }^{11}$ The areas for the observed three bilayer morphologies in the phase separation region are differentiated by colors. The phase separation region is close to the DPPC-rich side. Note that the phase separation region is similar to that in the DOPC/DPPC/cholesterol system. ${ }^{6}$

When phase separation occurs, the distribution of the TD to the DOPC-rich domain and the DPPC-rich domain can be inferred from the WAXD measurements. Since the phase separation occurs when the fractions of DOPC and DPPC are the same (Fig. 4), we compared the WAXD results for DOPC:DPPC:TD $=x: x: 100-2 x$ with the DPPC/TD binary system. Figure 5 shows the lattice constant ${ }^{23} \mathrm{~s}$ and the corresponding chain-chain distance $d_{c}=(2 / \sqrt{3})$ s of the ordered hydrocarbon chains in the ordered gel phase domain. In both systems, s decreases with increasing TD fraction, which means that the molecular packing in the ordered gel phase domain is enhanced by the addition. The decrease in $s$ for the DOPC/DPPC/TD system is almost similar to that of the DPPC/TD system, if $s$ is depicted against the TD fraction in the whole system as shown in Fig. 5. Because the packing of hydrocarbon chains only in the DPPC-rich domain is sensed by WAXD, the similar decrease in $s$ indicates that the amount of TD in the DOPC-rich and DPPC-rich domains is almost equal. (For example, for DOPC:DPPC:TD $=40: 40: 20$, $10 \mathrm{~mol} . \%$ of TD is in the DOPC-rich phase and $10 \mathrm{~mol} . \%$ residue is in the DPPC-rich phase. Then, DPPC:TD $=40: 10$ $=80: 20$.) The almost equal amount of TD in the DOPC-rich and DPPC-rich domains indicates that the tie line in the phase-separated region in the phase diagram is nearly parallel to the DOPC-DPPC baseline of the diagram, as shown in Fig. 4(b).

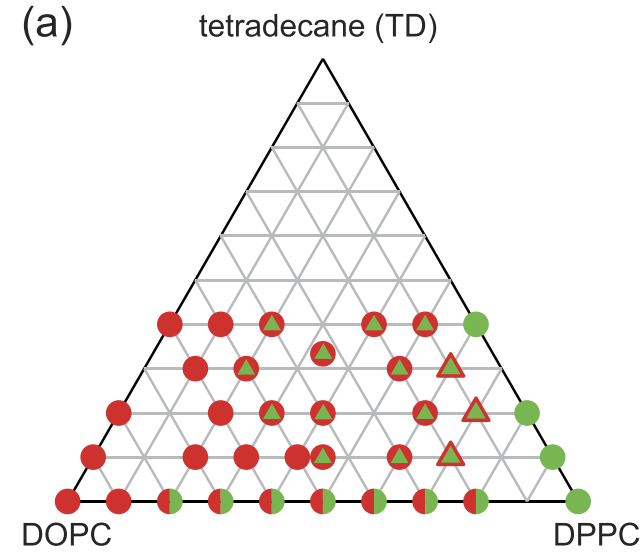

(b) tetradecane (TD)

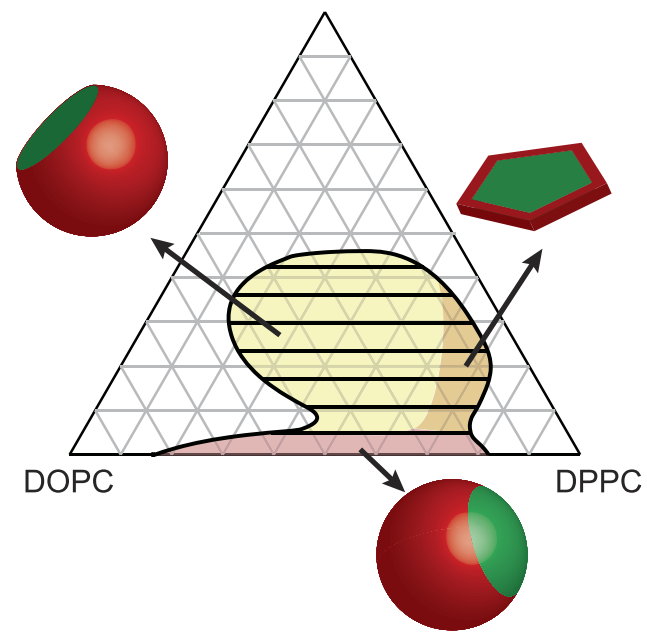

FIG. 4. (a) Summary of experimental results for the DOPC/DPPC/TD system at room temperature. Red and green circles indicate the formation of homogeneous vesicles without phase separation, as in Fig. 2(b). Half-red/half-green circles indicate the ordinary phase separation of spherical vesicles as in Fig. 2(a). A green triangle in a red circle indicates a phase-separated rugged vesicle with the characteristic morphology, in which flat DPPC-rich domains are connected with DOPC-rich flexible domains, as in Figs. 1(a)-1(c). A green triangle in a red triangle indicates flat flakes of the DPPC-rich bilayer rimmed with DOPC-rich domains, as in Fig. 3. (b) Ternary phase diagram of the phase separation of the DOPC/DPPC/TD system at room temperature. The areas where the three bilayer morphologies are observed are differentiated by distinct colors in the phase separation region. Tie lines in the phase separation region are depicted with solid black lines, which was inferred from the wide-angle X-ray diffraction results (Fig. 5).

Finally, we briefly discuss the asymmetric emergence of the phase separation region. Since the distribution of TD is almost equal to the DOPC-rich and DPPC-rich domains, the system can approximately be regarded as a binary system of a DOPC/TD mixture and a DPPC/TD mixture. The phase separation of a binary system can be fundamentally described by a mean field approximation, ${ }^{24}$ in which the free energy $\mathrm{F}$ is expressed as 


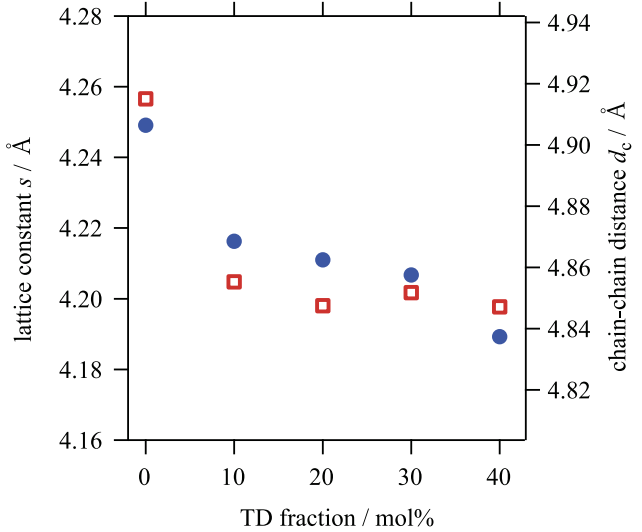

FIG. 5. Lattice constant $s$ and the corresponding chain-chain distance $d_{c}$ $=(2 / \sqrt{3}) \mathrm{s}$ of regularly ordered hydrocarbon chains in the DPPC-rich ordered phase against the TD fraction in the whole system. Red squares are for the DPPC/TD binary system, while blue circles are for the DOPC/DPPC/TD ternary mixture with the same fractions of DOPC and DPPC.

$$
\frac{F}{k_{\mathrm{B}} \mathrm{T}}=\phi_{\mathrm{A}} \log \phi_{\mathrm{A}}+\phi_{\mathrm{B}} \log \phi_{\mathrm{B}}+\chi \phi_{\mathrm{A}} \phi_{\mathrm{B}}
$$

where

$$
\chi=\frac{h}{\mathrm{~T}}\left(2 \epsilon_{\mathrm{AB}}-\epsilon_{\mathrm{AA}}-\epsilon_{\mathrm{BB}}\right),
$$

and $k_{\mathrm{B}}$ is Boltzmann's constant, $h$ is a positive constant, $\mathrm{T}$ is temperature, $\phi_{\mathrm{X}}$ is the fraction of component $\mathrm{X}, \epsilon_{\mathrm{AB}}$ is the pair interaction between $\mathrm{A}$ and $\mathrm{B}$, and $\epsilon_{\mathrm{AA}}$ and $\epsilon_{\mathrm{BB}}$ are the pair interactions between the same component, respectively. In this treatment, the binary system is assumed to be symmetric, i.e., the phase separation region is symmetric. ${ }^{25}$ In contrast, the observed phase separation region emerges asymmetrically close to the DPPC-rich side.

In the present case, the molecules form an ordered lattice in the DPPC-rich ordered gel phase. When an impurity is added to the ordered phase, the lattice structure is disturbed and the inter-molecular interactions are plausibly reduced. If we consider the DOPC/TD mixture as an impurity (component $\mathrm{B}$ ) in the ordered phase of the DPPC/TD mixture (component A), $\epsilon_{\mathrm{AA}}$ becomes dependent on the fraction, and it decreases with increasing $\phi_{\mathrm{B}}$ (the fraction of the DOPC/TD mixture). In this case, $\chi$ is simply expanded in terms of $\phi_{\mathrm{B}}$ as

$$
\begin{aligned}
\chi & =\frac{h}{\mathrm{~T}}\left[2 \epsilon_{\mathrm{AB}}-\epsilon_{\mathrm{AA}}\left(1-c \phi_{\mathrm{B}}\right)-\epsilon_{\mathrm{BB}}\right] \\
& =\frac{\mathrm{H}}{\mathrm{T}}\left(1+g \phi_{\mathrm{B}}\right),
\end{aligned}
$$

where $c$ and $g$ are positive constants. This is the simplest expansion of the Van Laar/Bragg-Williams model, ${ }^{25}$ which has been used empirically to describe asymmetric phase separation. Since $g$ is positive, the phase separation region is close to the DPPC-rich side, ${ }^{25}$ as observed in our experiments. Thus, the asymmetric phase separation is reasonable in the present case.

As stated above, DOPC/DPPC/cholesterol exhibits a similar phase separation region in the phase diagram. In this system, however, since the tie line is not parallel to the DOPCDPPC baseline, ${ }^{26}$ the phase separation cannot be regarded as a binary phase separation like the present system. The different affinities of cholesterol to the ordered phase and the disordered phase are probably the origin of the asymmetric phase separation. In short, the similar phase separation regions in these two systems are coincidental.

\section{SUMMARY}

In this paper, the ternary phase behavior of two lipids that have ordered gel and disordered liquid-crystalline phases, respectively, and a mixture with an $n$-alkane are reported. When we use long $n$-alkanes (TD or HD), we observed the characteristic vesicle morphologies, in which the rigid and flat domains in the ordered phases are connected by flexible domains in the disordered phase. When the fraction of the lipid in the disordered phase is small, the bilayer does not form vesicles, and the flat ordered-phase bilayer is rimmed with a lipid in the disordered phase. For a short n-alkane (DD), such characteristic morphologies were not observed due to weak enhancement of the molecular packing in the ordered-phase domain by the alkane. In the DOPC/DPPC/TD system, the distribution of $\mathrm{TD}$ is almost equal to the ordered- and disordered-phase domains. The phase separation in the DOPC/DPPC/TD system occurs asymmetrically in the ternary phase diagram, which is interpreted by the Van Laar/Bragg-Williams model of phase separation.

The effect of an $n$-alkane on the phase separation is completely different from that of cholesterol, due to their different molecular shapes. Similar characteristic morphology and phase separation to those reported here are expected in systems containing other linear and long chain molecules, such as alkanols and fatty acids, ${ }^{27}$ since a similar enhancement of the molecular packing in the ordered gel phase has been reported for these long molecules. ${ }^{15}$ The properties of the raft in a biomembrane is expected to be strongly affected by these linear and long molecules, some of which are found in real cells.

For a systematic understanding of the additive effects on the phase separation behaviors, further investigations are not required to use only linear molecules but also bulkier molecules, such as stilbene. ${ }^{12,13}$

\section{ACKNOWLEDGMENTS}

This work was supported in part by a Grant-in-Aid for Scientific Research from the Japan Society for the Promotion of Science to M.H. (Grant Nos. 24740289, 15K13546, and 18K03555). The WAXD experiments were performed under the approval of the Photon Factory Program Advisory Committee (Proposal Nos. 2015G604 and 2013G525).

\section{REFERENCES}

${ }^{1}$ K. Simons and E. Ikonen, Nature 387, 569 (1997).

${ }^{2}$ M. Luckey, Membrane Structural Biology (Cambridge University Press, New York, 2008).

${ }^{3}$ M. F. Hanzal-Bayer and J. F. Hancock, FEBS Lett. 581, 2098 (2007). 
${ }^{4}$ D. Lingwood and K. Simons, Science 327, 46 (2010)

${ }^{5}$ S. L. Veatch and S. L. Keller, Phys. Rev. Lett. 89, 268101 (2002).

${ }^{6}$ S. L. Veatch and S. L. Keller, Biophys. J. 85, 3074 (2003).

${ }^{7}$ T. P. W. McMullen and R. N. McElhaney, Biochim. Biophys. Acta 1234, 90 (1995).

${ }^{8}$ T. J. McIntosh, S. A. Simon, and R. C. MacDonald, Biochim. Biophys. Acta 597, 445 (1980).

${ }^{9}$ K. Lohner, Chem. Phys. Lipids 57, 341 (1991).

${ }^{10}$ M. Hishida, A. Endo, K. Nakazawa, Y. Yamamura, and K. Saito, Chem. Phys, Lipids 188, 61 (2015).

${ }^{11}$ M. Hishida, R. Yanagisawa, H. Usuda, Y. Yamamura, and K. Saito, J. Chem. Phys. 144, 041103 (2016).

${ }^{12}$ K. Nakazawa, M. Hishida, S. Nagatomo, Y. Yamamura, and K. Saito, Chem. Lett. 43, 1352 (2014).

${ }^{13}$ K. Nakazawa, Y. Kawata, M. Hishida, Y. Yamamura, and K. Saito, Chem. Lett. 47, 240 (2018).

${ }^{14}$ H. Usuda, M. Hishida, Y. Yamamura, and K. Saito, Langmuir 32, 5966 (2016).

${ }^{15}$ H. Usuda, M. Hishida, Y. Yamamura, and K. Saito, Chem. Lett. 47, 1512 (2018).
${ }^{16}$ D. A. Haydon, B. M. Hendry, S. R. Levinson, and J. Requena, Biochim. Biophys. Acta 470, 17 (1977).

${ }^{17}$ M. Weinrich and D. L. Worcester, J. Phys. Chem. B 117, 16141 (2013).

${ }^{18}$ J. P. Reeves and R. M. Dowben, J. Cell. Physiol. 73, 49 (1969).

${ }^{19}$ M. Hishida, H. Seto, and K. Yoshikawa, Chem. Phys. Lett. 411, 267 (2005).

${ }^{20} \mathrm{M}$. Hishida, H. Seto, N. L. Yamada, and K. Yoshikawa, Chem. Phys. Lett. 455, 297 (2008).

${ }^{21}$ N. Shimokawa, M. Hishida, H. Seto, and K. Yoshikawa, Chem. Phys. Lett. 496, 59 (2010).

${ }^{22}$ Y. Suezaki, H. Kamaya, and I. Ueda, Biochim. Biophys. Acta 818, 31 (1985).

${ }^{23}$ D. Marsh, Chem. Phys. Lipids 165, 59 (2011).

${ }^{24}$ R. A. L. Jones, Soft Condensed Matter (Oxford University Press, Oxford, 2002).

${ }^{25}$ R. Koningsveld, W. H. Stockmayer, and E. Nies, Polymer Phase Diagrams: A Textbook (Oxford University Press, Oxford, 2001).

${ }^{26}$ P. Uppamoochikkal, S. Tristram-Nagle, and J. F. Nagle, Langmuir 26, 17363 (2010).

${ }^{27}$ N. Shimokawa, R. Mukai, M. Nagata, and M. Takagi, Phys. Chem. Chem. Phys. 19, 13252 (2017). 